\title{
Fuzzy Ordering Relation and Fuzzy Poset
}

\author{
Branimir Šešelja and Andreja Tepavčevič ${ }^{\star}$ \\ Department of Mathematics and Informatics \\ Faculty of Sciences, University of Novi Sad \\ Trg D. Obradovića 4, 21000 Novi Sad, Serbia \\ seselja@im.ns.ac.yu, etepavce@eunet.yu
}

\begin{abstract}
Connections between (weakly) reflexive, antisymmetric and transitive lattice-valued fuzzy relations on a nonempty set $X$ (fuzzy ordering relations on $X$ ) and fuzzy subsets of a crisp poset on $X$ (fuzzy posets) are established and various properties of cuts of such structures are proved.

A representation of fuzzy sets by cuts corresponding to atoms in atomically generated lattices has also been given.
\end{abstract}

AMS Mathematics Subject Classification (1991): 04A72.

Keywords and Phrases: Lattice valued fuzzy ordering relation, fuzzy weak ordering relation, fuzzy poset, cutworthy approach.

\section{Introduction}

The present research belongs to the theory of fuzzy ordering relations. These are investigated as lattice valued structures. Therefore, the framework as well as the subject are within the order theory. Fuzzy structures presented here support cutworthy properties, that is, crisp fuzzified properties are preserved under cut structures. Therefore, the co-domains of all mappings (fuzzy sets) are complete lattices, without additional operations. Namely, such lattices support the transfer of crisp properties to cuts.

Due to the extensive research of fuzzy ordering relations, we mention only those authors and papers which are relevant to our approach. From the earlier period, we mention papers by Ovchinnikov (910], and papers cited there); we use his definition of the ordering relation. In the recent period, there are papers by Bělohlávek (most of the relevant results are collected in his book [1]). He investigates also lattice-valued orders, the lattice being residuated. Recent important results concerning fuzzy orders and their representations are obtained by De Baets and Bodenhofer (see a state-of-the-art overview about weak fuzzy orders, 4]).

\footnotetext{
* This research was partially supported by Serbian Ministry of Science and Environment, Grant No. 144011 and by the Provincial Secretariat for Science and Technological Development, Autonomous Province of Vojvodina, grant "Lattice methods and applications".
} 
Most of the mentioned investigations of fuzzy orders are situated in the framework of $T$-norms, or more generally, in the framework of residuated lattices and corresponding operations.

As mentioned above, our approach is purely order-theoretic.

Motivated by the classical approach to partially ordered sets, we investigate fuzzy orders from two basic aspects. Firstly we consider fuzzy posets, i.e., fuzzy sets on a crisp ordered set. The other aspect is a fuzzification of an ordering relation. As the main result we prove that there is a kind of natural equivalence among these two structures. Namely, starting with a fuzzy poset, we prove that there is a fuzzy ordering relation on the same set with equal cuts. Vice versa, for every fuzzy ordering relation there is a fuzzy poset on the same underlying set, so that cut-posets of two structures coincide. We also present some properties of cuts, which give an additional description of fuzzy orders.

Our results are partially analogue to those in paper [14, in which similar problems are discussed for fuzzy lattices. We mention that we use fuzzy weak ordering relations (reflexivity is weakened), which differs from the notion of the weak order used in the paper [4].

\section{Preliminaries}

We use well known notions from the classical theory of ordered structures (see e.g., 6]). A poset $(P, \leq)$ is a nonempty set $P$ endowed with an ordering relation (order) $\rho$, not necessarily linear. It is well known that an order on a given set by definition fulfills properties of reflexivity, antisymmetry and transitivity. We use also the notion of a weak ordering relation on a set $P$, which is an antisymmetric and transitive relation, satisfying also the weak reflexivity on $P$ : for any $x, y \in P$

if $x \rho y$ then $x \rho x$ and $y \rho y$.

Throughout the paper $L$ is a complete lattice with the top element (1) and the bottom element $(0)$; sometimes these are denoted by $1_{L}$ and $0_{L}$. An element $a \in L$ distinct from 0 is an atom in $L$ if it covers the least element 0 , i.e., if $0 \leq x \leq a$ implies $x=0$ or $x=a$. If there is a single atom in $L$ which is below all other non-zero elements in $L$, then it is called a monolith. A lattice $L$ is atomic if for every non-zero element $x \in L$, there is an atom $a$ such that $a \leq x$. Finally, a lattice $L$ is atomically generated if every non-zero element is a join (supremum) of atoms.

Next we present notions connected with lattice valued fuzzy sets and structures. For more details we refer to the overview articles 1112.

A fuzzy set on a nonempty set $S$ is a mapping $\mu: S \rightarrow L$. A cut set (briefly cut) of $\mu$ is defined for every $p \in L$, as the subset $\mu_{p}$ of $S$, such that $x \in \mu_{p}$ if and only if $\mu(x) \geq p$ in $L$. A strong cut of $\mu$ is a subset $\mu_{p}^{>}$of $S$ such that for every $p \in L, x \in \mu_{p}^{>}$if and only if $\mu(x)>p$ in $L$.

A property or a notion which is transferred or generalized to fuzzy structures is said to be cutworthy if it is preserved by all cuts of the corresponding fuzzy structure (see e.g., 8]). 
If $X$ is a nonempty set, any mapping $\rho: X^{2} \rightarrow L$ is an $L$-valued (lattice valued) relation on $X$.

Since a fuzzy relation is a fuzzy subset of $X^{2}$, a cut relation is a cut of the corresponding fuzzy set: for $p \in L$, a $p$-cut of $\rho$ is a subset $\rho_{p}$ of $X^{2}$, such that $\rho_{p}=\{(x, y) \mid \rho(x, y) \geq p\}$.

Now, we recall fuzzy ordering relations and some of their main properties. Among many definitions of fuzzy orders, we use the following, which, in our opinion, is the most natural lattice-valued generalization of the crisp order. The reason for this opinion is that all non-trivial cuts of such fuzzy orders are crisp ordering relations, i.e., its importance should be understood in the cutworthy sense.

An $L$-valued relation is

- reflexive if

$$
\rho(x, x)=1, \text { for every } x \in X ;
$$

- weakly reflexive if

$$
\rho(x, x) \geq \rho(x, y) \text { and } \rho(x, x) \geq \rho(y, x), \text { for all } x, y \in X \text {; }
$$

- antisymmetric if

$$
\rho(x, y) \wedge \rho(y, x)=0, \text { for all } x, y \in X, x \neq y
$$

- transitive if

$$
\rho(x, y) \wedge \rho(y, z) \leq \rho(x, z) \text {, for all } x, y, z \in X
$$

An $L$-valued relation $\rho$ on $X$ is an $L$-fuzzy ordering relation (fuzzy order) on $X$ if it is reflexive, antisymmetric and transitive.

An $L$-valued relation $\rho$ on $X$ is a weak $L$-fuzzy ordering relation (weak fuzzy order) on $X$ if it is weakly reflexive, antisymmetric and transitive.

Observe that, like in the crisp case, every fuzzy order is, at the same time, a fuzzy weak order on the same set, while the reverse need not be true.

Theorem 1. A relation $\rho: S^{2} \rightarrow L$ is an $L$-fuzzy ordering relation if and only if all cuts except 0 -cut are ordering relations.

Proof. Let $\rho: S^{2} \rightarrow L$ be a fuzzy ordering relation, and let $p \in L$. If $p=0$, then $\rho_{0}=S^{2}$ and it is not ordering relation unless $|S|=1$.

Let $p \neq 0$. Then, $(x, x) \in \rho_{p}$, by the reflexivity of $\rho$.

If $(x, y) \in \rho_{p}$ and $(y, x) \in \rho_{p}$, then $\rho(x, y) \geq p$ and $\rho(y, x) \geq p$ and hence $\rho(x, y) \wedge \rho(y, x) \geq p$, which is true only in case $x=y$.

If $(x, y) \in \rho_{p}$ and $(y, z) \in \rho_{p}$ then $\rho(x, y) \geq p$ and $\rho(y, z) \geq p$. Therefore $\rho(x, z) \geq \rho(x, y) \wedge \rho(y, z) \geq p$ and hence $(x, z) \in \rho_{p}$.

On the other hand, suppose that $\rho$ is a fuzzy relation such that all cuts (except 0 -cut) are ordering relations.

Since all $p$ cuts are reflexive relations, $\rho(x, x)=\bigvee_{p \in L} \rho_{p}(x, x)=1$. 
Further, let $x \neq y$ and let $\rho(x, y) \wedge \rho(y, x)=p$. Then, $(x, y) \in \rho_{p}$ and $(y, x) \in$ $\rho_{p}$, and by antisymmetry of $\rho_{p}$, we have that $p=0$.

Similarly, we can prove the transitivity of $\rho$.

\section{$3 \quad$ Fuzzy Posets and Fuzzy Orders}

In paper [14, fuzzy lattices are considered from two aspects: fuzzy lattices as fuzzy algebraic structures and fuzzy lattices as particular fuzzy ordered sets. In the same paper, conditions for transferring of one concept to another are given.

A fuzzy lattice as a fuzzy algebraic structure is obtained by a fuzzification of the carrier, while a fuzzy lattice as a fuzzy ordered structure is obtained by a fuzzification of the ordering relation.

In this part we formulate analogue connections for fuzzy orders.

\section{Fuzzy Poset}

If $(P, \leq)$ is a poset and $(L, \wedge, \vee)$ a complete lattice, we consider a fuzzy poset as a fuzzy set $\mu: P \rightarrow L$ with the given crisp ordering relation $\leq$.

Cut sets are sub-posets of $P$, i.e., every cut is a subset of $P$ endowed with the restriction of the relation $\leq$ to this subset. These restriction-relations can also be considered as weak orders on $P$. We denote these restriction-relations with the same symbol, $\leq$.

\section{Fuzzy Weak Order}

On the other hand, starting with the same poset $(P, \leq)$, we fuzzify a relation $\leq$ as a mapping from $P^{2} \rightarrow\{0,1\}$. Thus we obtain a fuzzy weak ordering relation on $P$, as a mapping from $P^{2}$ to $L$ which is weakly reflexive, antisymmetric and transitive.

It is straightforward to check that the cut sets of fuzzy weak order on $P$ are crisp weak orderings on $P$.

A connection between two fuzzifications (a fuzzy poset and a fuzzy weak order) is given in the next theorems.

Lemma 1. Let $\rho: S^{2} \rightarrow L$ be an $L$-fuzzy ordering relation, such that $L$ is a complete lattice having a monolith a. Then, the strong cut $\rho_{0}^{>}$is a crisp ordering relation on the set $S$.

Proof. Since $\rho(x, x)=1>0$, we have that $(x, x) \in \rho_{0}^{>}$.

If $\rho(x, y)>0$ and $\rho(y, x)>0$, we have that $\rho(x, y) \geq a$ and $\rho(x, y) \geq a$, hence $\rho(x, y) \wedge \rho(y, x) \geq a$ and thus $x=y$.

If $\rho(x, y)>0$ and $\rho(y, z)>0$, we have that $\rho(x, y) \wedge \rho(y, z) \geq a$ and hence by fuzzy transitivity, $\rho(x, z) \geq a$. Therefore $(x, z) \in \rho_{0}^{>}$.

Remark. The assumption that $L$ has a monolith is here essential. Indeed, the strong 0 cut in a fuzzy ordered set with the co-domain being a complete lattice without the unique atom need not be an ordered set. Example 1 below illustrates this fact.

Theorem 2. Let $\mu: P \rightarrow L$ be an $L$-fuzzy poset. Then, the mapping $\rho: P^{2} \rightarrow L$ defined by 


$$
\rho(x, y)= \begin{cases}1, & \text { if } x=y \\ \mu(x) \wedge \mu(y), & \text { if } x<y \\ 0, & \text { otherwise. }\end{cases}
$$

is an L-fuzzy ordering relation.

Proof. The mapping $\rho$ is reflexive by the definition. To prove the antisymmetry, assume $x \neq y$. Then, by the antisymmetry of $<$, if $x<y$, then it is not $y<x$ and vice versa. Therefore, at least one of the values $\rho(x, y)$ or $\rho(y, x)$ is 0 and thus $\rho(x, y) \wedge \rho(y, x)=0$.

We prove the transitivity. In case $\rho(x, y) \wedge \rho(y, z)=0$, it is true that $\rho(x, y) \wedge$ $\rho(y, z) \leq \rho(x, z)$. In case $\rho(x, y) \wedge \rho(y, z)>0$, we have that either $x=y$ or $y=z$ or $x<y$ and $y<z$. In case $x=y$ (similarly for $y=z$ ), we have that $\rho(x, y) \wedge \rho(y, z)=1 \wedge \rho(x, z) \leq \rho(x, z)$.

In case $x<y$ and $y<z$, we have $x<z$ and thus $\rho(x, y) \wedge \rho(y, z)=\mu(x) \wedge$ $\mu(y) \wedge \mu(y) \wedge \mu(z) \leq \mu(x) \wedge \mu(z)=\rho(x, z)$.

In the following we use the notation $0 \oplus L$ for a lattice $L^{\prime}$ obtained by adding a new least element 0 to the lattice $L$. The least element in $L$ becomes thus a monolith in $L^{\prime}$ and it is denoted by $0_{L}$ (see Figure 1 ).

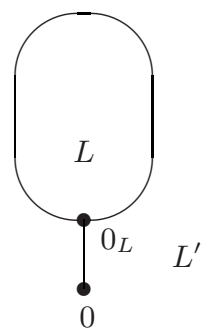

Fig. 1.

Theorem 3. Let $\mu: P \rightarrow L$ be an $L$-fuzzy poset and let $L^{\prime}=0 \oplus L$. Then, the mapping $\rho: P^{2} \rightarrow L^{\prime}$ defined by

$$
\rho(x, y)= \begin{cases}\mu(x) \wedge \mu(y), & \text { if } x \leq y \\ 0, & \text { otherwise. }\end{cases}
$$

is an $L$-fuzzy weak ordering relation. Moreover cuts $\mu_{p}$ and $\rho_{p}$, for $p \in L$ coincide as crisp sub-posets.

Proof. If $x \leq y$ then $\rho(x, x)=\mu(x) \geq \mu(x) \wedge \mu(y)=\rho(x, y)$. In case $x \not \leq y$, we have $\rho(x, y)=0$ and hence weak reflexivity is also satisfied.

The proof for antisymmetry is the same as in the previous theorem.

In the proof of transitivity, similarly as in the previous theorem, the only nontrivial case is when $\rho(x, y) \wedge \rho(y, z)>0$. Thus, $x \leq y$ and $y \leq z$, and hence $x \leq z$. Therefore, $\rho(x, z)=\mu(x) \wedge \mu(z) \geq \mu(x) \wedge \mu(y) \wedge \mu(y) \wedge \mu(z)=$ $\rho(x, y) \wedge \rho(y, z)$. 
Let $p \in L$. We prove that $\rho_{p}$ is a weak ordering relation on $P$, the same one which corresponds to $\mu_{p}$. As already mentioned, a weak ordering relation on $P$ is an ordering relation on a subset of $P$.

$(x, y) \in \rho_{p}$ if and only if $\rho(x, y) \geq p$ if and only if $\mu(x) \wedge \mu(y) \geq p$ if and only if $\mu(x) \geq p$ and $\mu(y) \geq p$ if and only if $x, y \in \mu_{p}$, completing the proof.

Next theorem gives the converse of the previous ones, and proves that every fuzzy ordering relation determines a fuzzy ordered set.

Theorem 4. Let $\rho: P^{2} \rightarrow L^{\prime}$ be an $L$-fuzzy weak ordering relation, where $L^{\prime}=$ $0 \oplus L$ is a complete lattice with a monolith $0_{L}$, the top element $1_{L}$ and the bottom element 0 . Let $S:=\{x \mid \rho(x, x)>0\}$. Then, the mapping $\mu: S \rightarrow L$ defined by:

$$
\mu(x)=\rho(x, x)
$$

is an $L$-fuzzy poset on $(S, \leq)$, where the order is the strong 0 -cut, i.e., $\leq=\rho_{0}^{>}$. In addition, for any $p \in L$, from $(x, y) \in \rho_{p}$ it follows that $x \in \mu_{p}$ and $y \in \mu_{p}$.

Proof. The relation $\rho_{0}^{>}$, by Lemma 1 , is a crisp ordering relation, which is denoted by $\leq . \mu$ is a fuzzy set on $P$, so $(P, \leq)$ is an $L$-fuzzy ordered set.

Let $p \in L$. Then from $(x, y) \in \rho_{p}$ it follows that $\rho(x, y) \geq p$ and hence $\rho(x, x) \geq p$ and $\rho(y, y) \geq p$. Therefore, $x \in \mu_{p}$ and $y \in \mu_{p}$.

In the following we present some properties of fuzzy ordering relations connecting them to crisp cut-ordering relations.

Proposition 1. Let $\rho: S^{2} \rightarrow L$ be an $L$-fuzzy weak ordering relation, where $L$ is an atomic lattice with the set of atoms $A$. Then, there is a family of posets $\left\{P_{a} \mid a \in A\right\}$ deduced from $\rho$ such that each poset in the family is a maximum poset under inclusion, being a cut of fuzzy relation $\rho$. In addition, every cut poset is a sub-poset of a poset in this family.

Proof. In Theorem 1 it is proved that any cut set, except 0-cut is a crisp weak ordering relation, or a crisp ordering relation on a subset of $S$. It is well known that from $p \leq q$ it follows that $\rho_{q} \subseteq \rho_{p}$. Since 0 -cut is not an ordering relation in a general case (it is equal to $S^{2}$ ), the maximum posets being the cut sets are $\rho_{a}$ for $a$ atoms. Since $L$ is atomic, by the same property we deduce that every cut except 0 is a subposet of a poset in this family.

$$
\begin{array}{c|ccccc}
\rho & a & b & c & d & e \\
\hline a & p & p & 0 & 0 & 0 \\
b & 0 & 1 & 0 & 0 & r \\
c & p & 1 & 1 & 0 & r \\
d & 0 & q & q & q & 0 \\
e & p & p & 0 & 0 & 1 .
\end{array}
$$

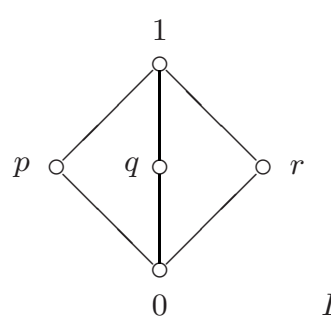

L

Fig. 2. 
Example 1. Let $\rho$ be a fuzzy weak ordering relation on set $S=\{a, b, c, d, e\}$, defined by the table, where the co-domain lattice is given in Figure 2.

Cuts of this fuzzy relation are as follows:

\begin{tabular}{|c|c|c|c|c|}
\hline & $a b c d e$ & $\rho_{p} \mid a b c d e$ & $\rho_{q} \mid a b c d e$ & $\rho_{r} \mid a b c d e$ \\
\hline$a$ & $\overline{000000}$ & \begin{tabular}{l|lllllll}
$a$ & 1 & 1 & 0 & 0 & 0
\end{tabular} & \begin{tabular}{l|llllll}
$a$ & 0 & 0 & 0 & 0 & 0
\end{tabular} & \begin{tabular}{l|llllll}
$a$ & 0 & 0 & 0 & 0 & 0
\end{tabular} \\
\hline$b$ & 01000 & \begin{tabular}{l|lllll}
$b$ & 0 & 1 & 0 & 0 & 0
\end{tabular} & 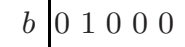 & \begin{tabular}{l|lllll}
$b$ & 0 & 1 & 0 & 0 & 1
\end{tabular} \\
\hline$c$ & $\begin{array}{lllll}0 & 1 & 1 & 0 & 0\end{array}$ & 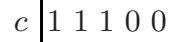 & 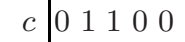 & \begin{tabular}{c|lllll}
$c$ & 0 & 1 & 1 & 0 & 1
\end{tabular} \\
\hline$d$ & $\begin{array}{lllll}0 & 0 & 0 & 0 & 0\end{array}$ & $d \mid \begin{array}{llllll}d & 0 & 0 & 0 & 0\end{array}$ & $d \mid \begin{array}{lllll}0 & 1 & 1 & 1 & 0\end{array}$ & $d \mid \begin{array}{lllll}0 & 0 & 0 & 0 & 0\end{array}$ \\
\hline$e$ & $\begin{array}{llllll}0 & 0 & 0 & 0 & 1\end{array}$ & 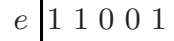 & $e \quad \begin{array}{lllll}0 & 0 & 0 & 0 & 1\end{array}$ & $\left.e \mid \begin{array}{lllll}0 & 0 & 0 & 0\end{array}\right]$ \\
\hline
\end{tabular}

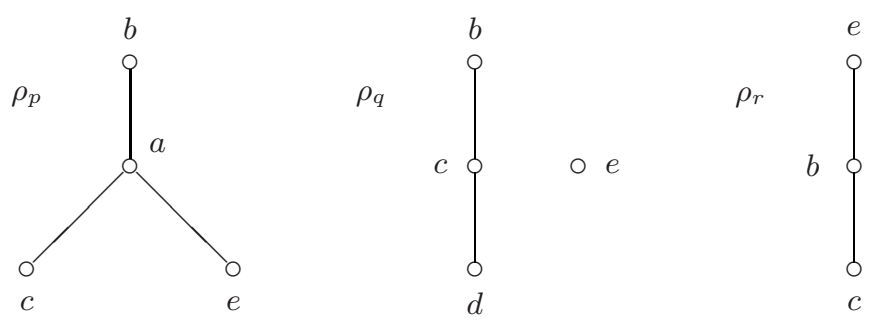

Fig. 3.

Considering three cuts that corresponds to atoms of $L$, we can see that posets corresponding to other cuts are sub-posets of some of these (in this case only $\left.\rho_{1}\right)$ (Figure 3).

Proposition 2. Let $\rho: S^{2} \rightarrow L$ be an $L$-fuzzy weak ordering relation, where $L$ is an atomic lattice with the set of atoms $A$. Then $\rho(x, y)>0$ if and only if there is an $a \in A$, such that $x \leq y$ in $P_{a}$, where $\left\{P_{a} \mid a \in A\right\}$ is a family of posets defined in the previous proposition.

Proof. If $\rho(x, y)=p>0$, for a $p \in L$, then there is an atom $a \in A$, such that $a \leq p$ and thus $x \leq y$ in $P_{a}$.

To prove the converse, if there is an $a \in A(a \neq 0)$ such that $x \leq y$ in $P_{a}$, then $\rho(x, y) \geq a$ and hence $\rho(x, y)>0$.

Proposition 3. If $\rho: S^{2} \rightarrow L$ is an $L$-fuzzy weak ordering relation, where $L$ is an atomically generated lattice with the set of atoms $A$, then

$$
\rho(x, y)=\bigvee_{a \in A} a \cdot P_{a}(x, y),
$$

where $P_{a}(x, y)=1$ if $x \leq y$ in $P_{a}$ and 0 otherwise, $a \cdot 1=a$ and $a \cdot 0=0$.

Proof. Let $\rho(x, y)=p$ and let $p$ be the supremum of some atoms $\left\{a_{i} \mid i \in I\right\}$. The proposition is a direct consequence of the fact that $p$ is the supremum exactly of the atoms below it. 


\section{Conclusion}

There are many possibilities to fuzzify and investigate fuzzy ordering relations and fuzzy posets. It turns out that the approach presented here is very convenient for the cutworthy approach. Indeed, we have established a connection among fuzzy posets as fuzzy subsets of crisp posets and fuzzy orderings, so that cut sets are preserved. Therefore, depending of the concrete situation in which fuzzy order appears, it is possible to use both, fuzzy poset and fuzzy ordering: cut sets would be the same.

In addition, last propositions point out the importance of atoms in atomically generated lattice in representation of values of fuzzy sets by cuts corresponding to atoms.

\section{References}

1. Bělohlávek, R.: Fuzzy Relational Systems. Kluwer Academic Publishers, Dordrecht (2002)

2. Bodenhofer, U.: A New Approach to Fuzzy Orderings. Tatra Mt. Math. Publ. 16(Part I), 21-29 (1999)

3. Bodenhofer, U.: Representations and constructions of similarity based fuzzy orderings. Fuzzy Sets and Systems 137, 113-136 (2003)

4. Bodenhofer, U., De Baets, B., Fodor, J.: A compendium of fuzzy weak orders: Representations and constructions. Fuzzy Sets and Systems 158, 811-829 (2007)

5. Gorjanac-Ranitovic, M., Tepavčević, A.: General form of lattice-valued fuzzy sets under the cutworthy approach. Fuzzy Sets and Systems 158, 1213-1216 (2007)

6. Davey, B.A., Pristley, H.A.: Introduction to Lattices and Order. Cambridge University Press, Cambridge (1992)

7. Janis, V., Šeselja, B., Tepavčević, A.: Non-standard cut classification of fuzzy sets. Information Sciences 177, 161-169 (2007)

8. Klir, G., Yuan, B.: Fuzzy sets and fuzzy logic. Prentice Hall P T R, New Jersey (1995)

9. Ovchinnikov, S.V.: Similarity relations, fuzzy partitions, and fuzzy orderings. Fuzzy Sets an Systems 40(1), 107-126 (1991)

10. Ovchinnikov, S.V.: Well-graded spaces of valued sets. Discrete Mathematics 245, 217-233 (2002)

11. Šeselja, B., Tepavčević, A.: Completion of Ordered Structures by Cuts of Fuzzy Sets, An Overview. Fuzzy Sets and Systems 136, 1-19 (2003)

12. Šeselja, B., Tepavčević, A.: Representing Ordered Structures by Fuzzy Sets. An Overview, Fuzzy Sets and Systems 136, 21-39 (2003)

13. Šeselja, B.: Fuzzy Covering Relation and Ordering: An Abstract Approach, Computational Intelligence. In: Reusch, B. (ed.) Theory and Applications, pp. 295-300. Springer, Heidelberg (2006)

14. Tepavčević, A., Trajkovski, G.: L-fuzzy lattices: an introduction. Fuzzy Sets and Systems 123, 209-216 (2001) 